\title{
The Role of TLR and Chemokine in Wear Particle-Induced Aseptic Loosening
}

\author{
Qiaoli Gu, Qin Shi, and Huilin Yang \\ Department of Orthopaedic Surgery, The First Affiliated Hospital of Soochow University, Orthopaedic Institute of Soochow University, \\ 188 Shizi Road, Jiangsu, Suzhou 215006, China \\ Correspondence should be addressed to Huilin Yang, suzhouspine@163.com
}

Received 31 July 2012; Revised 2 October 2012; Accepted 3 October 2012

Academic Editor: Mouldy Sioud

Copyright ( 2012 Qiaoli Gu et al. This is an open access article distributed under the Creative Commons Attribution License, which permits unrestricted use, distribution, and reproduction in any medium, provided the original work is properly cited.

Wear particle-induced periprosthetic osteolysis remains the principal cause of aseptic loosening of orthopaedic implants. Monocytes/macrophages phagocytose wear particles and release cytokines that induce inflammatory response. This response promotes osteoclast differentiation and osteolysis. The precise mechanisms by which wear particles are recognized and induce the accumulation of inflammatory cells in the periprosthetic tissue have not been fully elucidated. Recent studies have shown that toll-like receptors (TLRs) contribute to the cellular interaction with wear particles. Wear particles are recognized by monocytes/macrophages through TLRs coupled with the adaptor protein MyD88. After the initial interaction, wear particles induce both local and systemic migration of monocytes/macrophages to the periprosthetic region. The cellular migration is mediated through chemokines including interleukin-8, macrophage chemotactic protein-1, and macrophage inhibitory protein1 in the periprosthetic tissues. Interfering with chemokine-receptor axis can inhibit cellular migration and inflammatory response. This paper highlights recent advances in TLR, and chemokine participated in the pathogenesis of aseptic loosening. A comprehensive understanding of the recognition and migration mechanism is critical to the development of measures that prevent wear particle-induced aseptic loosening of orthopaedic implants.

\section{Introduction}

Total joint replacement (TJR) by the implantation of indwelling prostheses is an effective operation in terms of relieving pain and restoring function. The common longterm complication of TJR is loosening of an artificial joint that requires revision surgery [1-3]. Kurtz et al. have shown that total hip and total knee revisions will increase by $137 \%$ and $601 \%$, respectively, from 2005 to 2030 in the United States [4]. In most cases, aseptic loosening is responsible for revision total joint replacement. It has been reported that aseptic loosening accounts for $70 \%$ of hip revisions and $44 \%$ of knee revisions $[5,6]$.

The dominant theory about the causes of aseptic loosening is the particle disease theory [7-9]. Particles can be generated as a result of wear. The concentration of wear particles is directly related to the amount of osteolysis. There are a great number of wear particles in the periprosthetic membrane between bone and prosthesis. These wear particles which are biologically active and indigestible can initiate an innate inflammatory reaction [10-12]. Actually, wear particles alter the function of numerous cells including monocytes, macrophages, fibroblasts, osteoblasts, osteoclasts, and mesenchymal stem cells (MSCs). Macrophages have been accepted to be the key target of wear particles. Wear particles can induce the proliferation, differentiation, and activation of macrophages [13, 14]. Upon activation, macrophages secrete a series of inflammatory cytokines including tumor necrosis factor- $\alpha$ (TNF- $\alpha$ ), interleukin- $\alpha$ (IL- $1 \alpha)$, IL- $1 \beta$, IL-6, and IL-8. These inflammatory mediators can induce osteoclast differentiation or inhibit osteoblast differentiation, leading to periprosthetic bone resorption $[15,16]$. Moreover, some cytokines can attract and recruit large numbers of cells including macrophages, osteoclasts, and lymphocytes to the local site. These recruited cells then produce more cytokines, and a progress perpetuates a cycle of inflammatory response [17-19]. Apart from macrophages, osteoblasts and fibroblasts can also phagocytose particles, which significantly 
increase the expression of TNF- $\alpha$, IL-6, and RANKL [20, 21]. It has recently been shown that MSCs participate in wear particle-induced aseptic loosening. MSCs are identified around the joint replacement and contribute to maintaining osseous tissue integrity [22]. Wear particles can induce the production of inflammatory cytokine of MSCs on the one hand, while inhibit osteogenic activity on the other hand, resulting in osteolysis in periprosthetic region $[23,24]$.

Although numerous studies have demonstrated the events underlying periprosthetic inflammation and osteolysis, there are still more questions. First, the mechanisms of the initial cellular interaction with wear particles and the subsequent inflammatory mediator production remain unknown. Second, wear particles induce not only a local response but also a systemic reaction. The mechanism of cellular migration induced by wear particles needs further clarification. In 2007, Takagi et al. first reported that tolllike receptors (TLRs) were detected in the tissues around aseptically loosened implants [25]. TLR-deficient mice displayed decreased osteolysis. There is increasing evidence that TLRs play a critical role in initiating cellular interaction with particles and the subsequent inflammatory cascade [26, 27]. Lassus et al. reported that chemokines participated in the cellular migration in response to wear particles $[28,29]$. On the basis of these findings, this paper will discuss the role of TLRs in the recognition of wear particles and the role of chemokines in the cellular migration induced by wear particles, respectively. We believe that this could provide valuable insight into the design of preventive and therapeutic strategies in the future.

\section{TLRs and Wear Particle-Induced Aseptic Loosening}

2.1. TLRs and Ligands. TLRs belong to a class of pattern recognition receptors that enable the innate immune system to distinguish self- from nonself-structure. Thirteen TLRs have been identified in mammals since 1997. TLR1, TLR2, TLR4, TLR5, TLR6, TLR10, and TLR11 are expressed on the cell surface, whereas TLR3, TLR7, TLR8, and TLR9 are expressed intracellularly on endosomal membranes [30]. They are type I transmembrane proteins composed of an intracellular toll/interleukin-1 receptor (TIR) domain and leucine-rich repeat motifs in the extracellular domain. Leucine-rich repeats recognize danger signals, while TIRs recruit adaptor proteins and mediate downstream signaling $[31,32]$. TLRs can recognize a myriad of stimuli including pathogen-associated molecular patterns (PAMPs) and damage-associated molecular patterns (DAMPs). PAMPs are exogenous molecules derived from bacteria, virus, and fungi. DAMPs include endogenous intracellular molecules and extracellular matrix. The intracellular molecules, such as heat-shock protein (HSP) and high mobility group protein 1 (HMGB1), are released into the extracellular milieu by necrotic cells and activated leukocytes. The extracellular matrix includes biglycan, tenascin- $\mathrm{C}$, and hyaluronic acid which are released upon injury and noninfectious inflammatory response. The PAMPs and DAMPs identified for each TLR member are listed in Table $1[30,33]$.
2.2. TLR Signaling and Negative Regulators. Ligand-TLR interactions trigger the recruitment of adaptor proteins through the cytoplasmic TIR domain. So far, five TIR-containing adaptors have been identified: myeloid differentiation primary response gene 88 (MyD88), TIR domain-containing adaptor protein (TIRAP)/Mal, TIR domain-containing adaptor protein-inducing IFN- $\beta$ (TRIF)/TICAM1, TRIFrelated adaptor molecule (TRAM)/TICAM2, and Sterile- $\alpha$ and HEAT-armadillo motifs containing protein (SARM) [34, 35]. Depending on the usage of adaptor molecules, the signaling pathways activated by TLR are divided into MyD88dependent and MyD88-independent pathways. MyD88 is the main adaptor shared by TLR1, TLR2, TLR4, TLR5, TLR6, TLR7, TLR8, TLR9, and TLR11. TIRAP/Mal is involved in the MyD88-dependent pathway via TLR2 and TLR4. The MyD88-dependent pathways lead to the activation of nuclear factor $(\mathrm{NF})-\kappa \mathrm{B}$ and activating protein-1 (AP-1), which is responsible for the production of proinflammatory cytokines such as TNF- $\alpha$, IL-1, and IL-12. MyD88independent pathways are mediated by TRIF or TRAM. TRIF is an important adaptor which is used by TLR3. TRAM can link the TIR domain of TLR4 with TRIF. The MyD88independent pathways lead to the activation of NF- $\kappa \mathrm{B}, \mathrm{AP}-$ 1 , and interferon-regulatory factors (IRFs). The activation of transcription factors finally triggers the production of cytokines including TNF- $\alpha$, IL- $1 \beta$, IL-6, and IFN- $\alpha / \beta / \gamma$.

TLR signaling mediates inflammatory responses which are important for host defense. However, inappropriate TLR signaling may be responsible for the pathogenesis of autoimmune diseases, chronic inflammatory diseases, and aseptic inflammatory diseases [36]. To avoid harmful and excessive inflammatory responses, the immune system has developed multiple mechanisms to control TLR signaling. Many negative regulators have been reported. According to the regulatory mechanisms, these negative regulators can be classified into three groups [37]: (1) negative regulators for degradation of signal proteins: TAG, SARM, IRF4, TIPE2, NLRX1, NLRC5, TANK, MSK1, MSK2, TAK1, SHP1, SHP2, A20, CYLD, USP4, and DUBA; (2) negative regulators for degradation of signal proteins: PDLIM2, Trim $30 \alpha$, and TRIM38; (3) negative regulators for transcriptional control: ATF3, IkBNS, Bcl-3, Nurr1, Ah receptor, Zc3h12a, TTP, miR155, miR-146a, and miR-21.

2.3. TLRs and Aseptic Loosening. TLRs have been observed on variety of cells including monocytes/macrophages, lymphocytes, fibroblasts, osteoblasts, and osteoclasts. It has recently been reported that TLRs were found in periprosthetic tissues of patients with aseptic loosening. The TLRpositive cells are dominantly monocyte/macrophages [25, 38, 39]. Moreover, both PAMPs and DAMPs have been indicated in the activation of TLRs in aseptic loosening [40, 41]. These findings suggested that TLRs may play an important role in the pathogenesis of aseptic loosening. We will now mainly discuss TLR1/2 and TLR 4 which have attracted more attention than other TLRs.

TLR 1 and TLR2 are expressed on the cell surface and can form heterodimer [33]. They participate in the recognition of extracellular microbial pathogenic components including 
TABLE 1: Toll-like receptors and their corresponding ligands.

\begin{tabular}{|c|c|c|}
\hline TLR & Exogenous ligands (PAMPs) & Endogenous ligands (DAMPs) \\
\hline TLR1 & $\begin{array}{l}\text { Triacyl lipopeptide } \\
\text { Soluble factors }\end{array}$ & $\beta$-defensin 3 \\
\hline $\begin{array}{l}\text { TLR2 } \\
\text { (dimerization } \\
\text { with TLR1or 6) }\end{array}$ & $\begin{array}{l}\text { Lipoglycans (mycobacterium) } \\
\text { Lipoteichoic acids (gram-positive bacteria) } \\
\text { Peptidoglycan (gram-positive bacteria) } \\
\text { Zymosan (yeast) }\end{array}$ & $\begin{array}{l}\text { HSP 60, HSP70, gp96, } \beta \text {-defensin 3, HMGB1, surfactant proteins, } \\
\text { HMGB1-nucleosome complexes, serum amyloid A, } \\
\text { eosinophil-derived neurotoxin, antiphospholipid antibodies, } \\
\text { cardiac myosin, PAUF, CEP, monosodium urate crystals, Biglycan, } \\
\text { versican, hyaluronic acid fragments }\end{array}$ \\
\hline TLR3 & dsRNA & mRNA \\
\hline TLR4 & $\begin{array}{l}\text { LPS (gram-negative bacteria) } \\
\text { Mannan (Candida) } \\
\text { Envelope protein (virus) } \\
\text { Hsp70 (exogenous) }\end{array}$ & $\begin{array}{l}\text { HMGB1, surfactant proteins, } \beta \text {-defensin 2, HSP60, HSP70, HSP72, } \\
\text { HSP22, Gp96, S100A8, S100A9, neutrophil elastase, antiphospholipid } \\
\text { antibodies, lactoferrin, serum amyloid A, oxidized LDL, saturated } \\
\text { fatty acids, resistin, PAUF, monosodium urate crystals, Biglycan, } \\
\text { fibronectin EDA, fibrinogen, tenascin C, Heparin sulphate fragments, } \\
\text { Hyaluronic acid fragments }\end{array}$ \\
\hline TLR5 & Flagellin (gram-negative bacteria) & Undetermined \\
\hline TLR6 & $\begin{array}{l}\text { Diacylpolypeptide } \\
\text { Lipoteichoic acid (gram-positive bacteria) }\end{array}$ & Undetermined \\
\hline TLR7/8 & ss RNA (virus) & Antiphospholipid antibodies, cardiac myosin, ss RNA, \\
\hline TLR9 & CpG motif (bacteria, virus) & IgG-chromatin complexes, mitochondrial DNA \\
\hline TLR10 & Diacylated peptide? & Immunostimulatory $\mathrm{CpG}$ motifs \\
\hline TLR11 & Profilin-like molecule & Undetermined \\
\hline
\end{tabular}

lipoprotein/lipopeptides, peptidoglycan, and atypical LPS [34]. When mouse femur was inserted with stainless steel rod and titanium particles, the expressions of TLR1 and TLR2 were found in peri-implant and bone tissues. RAW 264.7 cells expressed both TLR1 and TLR2. However, only TLR1 was increased when cultured with titanium particles [42]. TLR2 was detected on monocytes/macrophages in aseptic synovial-like membranes from loose implants [43]. Hirayama et al. found that the expression of TLR2 was markedly increased after stimulation with LPS-coated titanium particles. On the contrary, other TLRs such as TLR4, TLR5, and TLR9 were decreased, suggesting a self-protective mechanism after stimulation with LPS-coated titanium particles [26]. Using a murine calvarial model of particleinduced osteolysis, Greenfield et al. found the TLR2 ${ }^{-/-}$mice displayed more limited osteolysis than wild mice. TLR2 ${ }^{-/-}$ macrophages secreted reduced TNF- $\alpha$ when challenged with titanium particles. These in vitro and in vivo data strongly support the critical role of TLR $1 / 2$ in aseptic loosening of implants [41].

TLR4 is the membrane receptor that can recognize LPS, mannan, glycoinositolphospholipids, envelope proteins, or some self-proteins including HSP60 and HSP70 [44, 45]. As a receptor for LPS, TLR4 has received the most attention in aseptic loosening. There was a significant increase of TLR4 in the tissue around loosened replacement implants $[25,43,46,47]$. The mutation of TLR4 resulted in inhibited inflammatory response and osteolysis when exposed to wear particles [48]. TLR4 ${ }^{-/-}$mice displayed decreased osteolysis. These findings indicated that TLR4 played a key role in the pathogenesis of aseptic loosening. Monocytes/macrophages are equipped with TLRs and HSPs. Hao et al. found that
UHMWPE particle upregulated the expressions of TLR4 and HSP60 on monocytes. HSP60 can bind to TLR4, leading to the production of inflammatory cytokines such as IL- $1 \beta$, IL6 , and TNF- $\alpha$ [40]. In this scenario, TLR4 played a critical role because interfering with TLR4 resulted in reduced cytokine production [40]. Like UHMWPE particle, titanium particle exposure can also elicit cytokine production and osteolysis. This phenomenon resulted from the engagement of TLR4 and wear particles with adherent LPS. [48]. LPS can be detected in the tissues around aseptically loosened implants, which contributed to the inflammatory responses induced by wear particles [49]. However, it is still unclear whether endotoxin is required for the biological response to that wear particles. People found wear particles with LPS decreased the mRNA expression of TLR4 compared to wear particles without LPS. This can be explained by a self-protective mechanism. LPS-coated wear particles can be easily identified by macrophages via TLR4. After the initiation of response, TLR4 was downregulated to prevent excessive harmful host response [26]. The reduced expression of TLR4 mRNA was also found in RAW 264.7 cells or rat macrophages stimulated with titanium particles in vitro $[25,42]$. It seemed that auto- or paracrine inflammatory cytokine downregulated the expression of TLR to avoid damage caused by excessive inflammatory responses [42]. Interestingly, TLR $4^{-/-}$macrophages showed similar levels of TNF- $\alpha$ compared to wild-type macrophages when challenged with wear particles. The unexpected results may be caused by the cells used in the experiments because a variety of cells expressed TLR4. Beidelschies et al. found that, in macrophages that lack TLR4 and TLR2, the section of TNF- $\alpha$ was completely neutralized when stimulated 
with wear particles. However, osteolysis in vivo was only partly inhibited. They supposed that early inflammatory response induced by particles was TLR dependent, while later osteolysis was just partially TLR dependent.

Ligand-TLR binding induces rearrangements of TIR domains and recruitment of adaptors (MyD88, TRIF, and TIRAP), triggering the activation of NF- $\kappa \mathrm{B}$. Pearl et al. found that the inflammatory responses induced by PMMA particles can be decreased using a MyD88 inhibitor. Similar results were found in $\mathrm{MyD} 88^{-/-}$macrophages stimulated with PMMA [27]. These findings strongly supported that particles can be recognized through TLR, partly dependent on MyD88 signaling pathway. Maitra et al. reported that UHMWPE particles activated TLR1/TLR2, leading to an inflammatory program mediated by NF- $\kappa \mathrm{B}$-signaling pathway [50]. It has been reported that the $\mathrm{p} 38$ and JNK signaling pathways can mediate wear particle-induced osteoclast differentiation in vitro. However, the relationship between specific TLRs and downstream p38 and JNK-signaling pathways remains unknown in wear particle-induced aseptic loosening. It is hypothesized that wear particles can be recognized by TLR, triggering downstream signaling pathway including AP-1 and $\mathrm{NF}-\kappa \mathrm{B}$, leading to inflammatory responses and osteolysis (Figure 1).

\section{Chemokines and Wear Particle-Induced Aseptic Loosening}

3.1. Chemokines and Chemokine Receptors. Chemokines are a group of small proteins with a crucial role in leukocyte migration and activation. These molecules can also affect cytokine secretion, apoptosis, phagocytosis, angiogenesis, and collagen production $[51,52]$. The chemokine family can be classified into four groups as CXC, CC, CX3C, and XC, based on the number and spacing of conserved cysteines. $\mathrm{CXC}, \mathrm{CC}$, and $\mathrm{CX} 3 \mathrm{C}$ chemokines contain four conserved cysteines, whereas $\mathrm{XC}$ chemokines contain two conserved cysteines. In the $\mathrm{CXC}$ and $\mathrm{CX} 3 \mathrm{C}$ chemokines, one (CXC) or three $(\mathrm{CX} 3 \mathrm{C})$ amino acids are inserted between the first two of four cysteines. In the CC chemokines, the first two cysteines are adjacent. About 50 chemokines have been identified in humans. Most chemokines are members of CC or CXC groups, while others belong to $\mathrm{XC}$ or $\mathrm{CX} 3 \mathrm{C}$ groups $[53,54]$.

The effects of chemokines are mediated by a family of $G$ protein-coupled receptor on the target cell surface [55]. These chemokine receptors are also classified into four groups as CXCR, CCR, CX3R and XCR, based on the chemokine group they bind. About 25 chemokine receptors have been identified in human. The relationship between chemokines and chemokine receptors is complex because chemokine receptors bind different chemokines and vice versa.

3.2. Chemokines in Particle-Induced Cellular Migration. In the early time, it was assumed that wear particles induced a localized response. In brief, wear particles stimulated resident cells (including macrophages, osteoblast) to produce cytokines such as TNF- $\alpha$, IL- $1 \alpha$, IL- $1 \beta$, and IL-6,

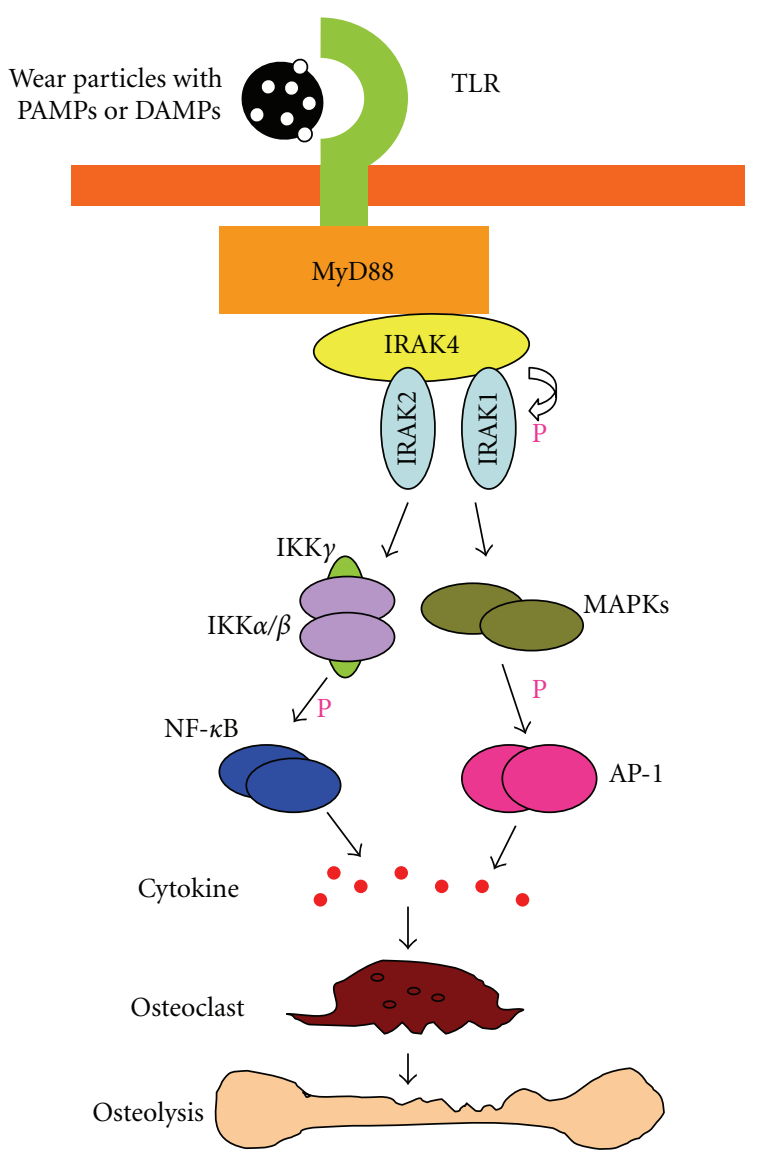

Figure 1: Wear particles induced TLR signal pathway. TLRs recognize wear particles with adherent PAMPs or DAMPs via MyD88. The binding of TLR and MyD88 phosphorylates IRAK4 which in turn phosphorylates IRAK1. The activation of AP-1 and NF- $\kappa \mathrm{B}$ leads inflammatory cytokine production and osteoclast differentiation which contribute to osteolysis.

resulting in local osteolysis. It has recently been shown that wear particles can induce chemokine expression in macrophages, fibroblasts, and osteoblasts, indicating a cellular migration mechanism in aseptic loosening [56-58]. Monocytes/macrophages accumulated in the periprosthetic tissue were mainly polarized M1 macrophages. Rao et al. hypothesized that monocyte/macrophage progenitors may be attracted to the local microenvironment in response to wear particles, and then differentiate into M1 phenotype [10]. More recently, Ren and Gibon both demonstrated that wear particles induced significant chemotaxis of macrophages in vivo [29, 59, 60]. These findings strongly support the mechanism of cellular migration to the site around the implants, which contributed to pathogenesis of aseptic loosening (Figure 2). In the following article we will focus on the specific chemokines involved in the cellular migration in response to wear particles.

IL-8, also known as CXCL8, is a member of the CXC chemokine family [61]. IL- 8 is mainly produced by monocytes/macrophages. The coupled receptors for IL- 8 are CXCR1 and CXCR2, which are located on the surface of 


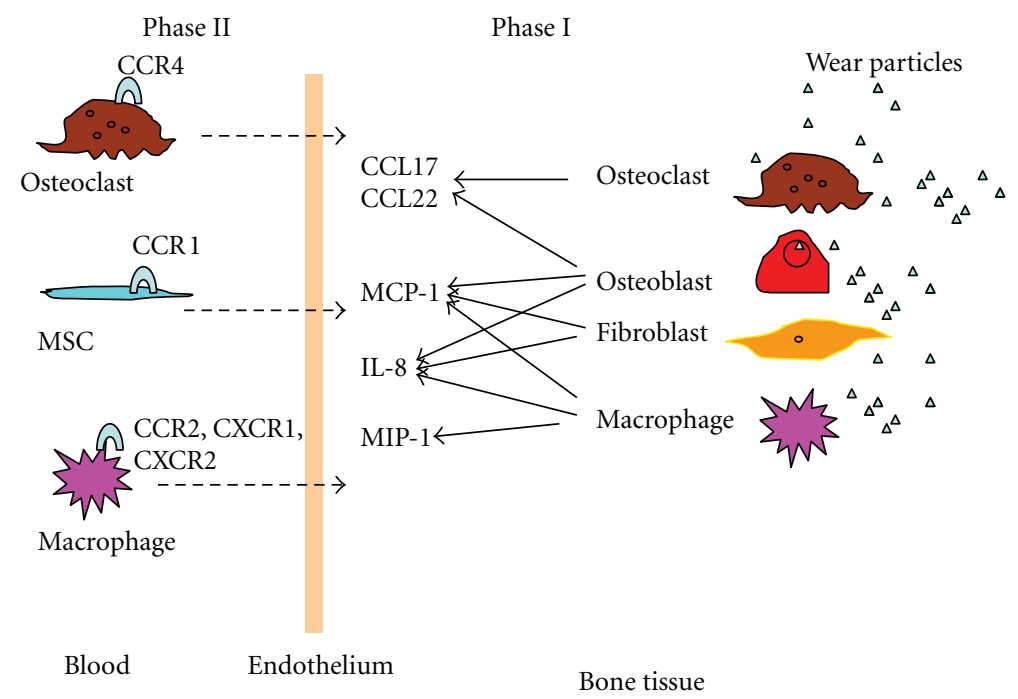

FIgURE 2: Wear particles induced chemokine expression and cellular migration.

macrophages, endothelial cells, mast cells, and epithelial cells [62]. The binding of IL-8 to receptors can trigger a series of biological effects including the activation and recruitment of neutrophils and macrophages [63]. Many reports have shown that IL-8 was upregulated in periprosthetic tissues $[28,64,65]$. These findings indicated that IL- 8 can be a marker of aseptic loosening. Actually, wear particles can stimulate the production of IL- 8 by MG- 63 and primary osteoblasts in vitro [66-70]. Kaufman et al. reported that primary human macrophages produced high level of IL-8 upon the stimulation of TiAlV particles [70]. UHMWPE and $\mathrm{CoCr}$ particles can also stimulate primary human macrophages to produce IL-8, although mildly compared to TiA1V particles. The latest research has shown that titanium particles can increase the production of IL- 8 by MSCs [24]. The increased production of IL- 8 can attract the migration of macrophages and osteoclasts to the site around implants, leading to osteolysis.

MCP-1, also known as CCL2, was identified based on its ability to chemoattract monocytes in vitro $[71,72]$. Further studies showed that MCP-1 can also attract memory $\mathrm{T}$ cells, natural killer cells, and macrophages to the sites of inflammation through the activation of CCR2 or CCR4 [73, 74]. It has been demonstrated that MCP-1 can be produced by endothelial cells, osteoblasts, fibroblasts, monocytes, and macrophages $[75,76]$. Wear particles can increase the expression of MCP-1 in primary human macrophages, MG-63 cells, and fibroblasts, resulting in the recruitment of monocytes/macrophages $[57,58,70,77]$. Increased expression of MCP-1 was also displayed in tissues from patients with failed arthroplasties, indicating that MCP-1 may be a potential marker of osteolysis [57, 78]. Huang et al. showed that PMMA or UHMWPE particles increased MCP-1 expression in RAW 264.7 cells. Supernatant from particle-stimulated RAW 264.7 cells displayed increased chemotactic response in THP-1 cells, which can be mitigated by neutralizing antibody to MCP-1 [79]. Interestingly, blocking CCR2 receptor reduced $\mathrm{PMMA}$-induced THP-1 cell migration, while has no effect on UHMWPE-induced THP-1 cell migration. Since most studies mentioned above are operated in vitro, it is impossible to understand whether the cells responding to wear particles are motivated locally or systemically. To clarify this issue, Gibon et al. injected MCP-1 into femur in a murine femoral implant model. They found that MCP-1 recruited exogenous RAW 264.7 cells to the femur upon the stimulation of UHMWPE particles. Blocking the interaction of MCP-1/CCR2 resulted in decreased migration of RAW 264.7 cells. MCP-1 also recruited primary murine macrophages into femur upon the stimulation of UHMWPE particles. Moreover, the recruitment of primary macrophages was lower when CCR2-deficient macrophages were injected [29]. These findings indicated that wear particle-induced MCP1 expression was critical to the migration of macrophages and subsequent inflammatory responses. The interruption of MCP-1/CCR2 axis may be a useful strategy to inhibit osteolysis.

MIP-1 includes MIP- $1 \alpha$ (CCL3) and MIP-1 $\beta$ (CCL4). It is mainly produced by lymphocytes, monocytes, macrophages, fibroblasts, and epithelial cells [80]. MIP- $1 \alpha$ played an important role in the migration of T cells, B cells, monocytes, dendritic cells, neutrophils, and natural killer cells [81]. The biological effects of MIP- $1 \alpha$ were mediated through their engagement with CCR1, CCR4, and CCR5 [51]. Like MCP-1, the expression of MIP- $1 \alpha$ was found in the periprosthetic tissues. Titanium and PMMA particles upregulated the production of MIP- $1 \alpha$ in primary human monocytes/macrophages, leading to increased migration of human monocyte. Neutralizing antibody to MIP- $1 \alpha$ mitigated the wear particleinduced migration [57]. These findings demonstrated that cellular migration mediated by MIP- $1 \alpha$ was important in 
wear particle-stimulated inflammatory responses. However, in another research, MIP- $1 \alpha$ seemed not to play a role in the chemotaxis function of wear particle-challenged RAW 264.7 cells. First, RAW 264.7 cells produced similar levels of MIP$1 \alpha$ when stimulated with or without wear particles. Second, although MIP- $1 \alpha$ possessed potent chemotactic ability for macrophages, neutralizing antibody to MIP- $1 \alpha$ failed to inhibit the migration of THP- 1 cells in the culture stimulated with particles [79]. These results are complicated and have not been explained by now. One possible explanation may be the specific culture condition or neutralizing antibody used in the experiments. Surprisingly, the supernatant from PMMA-challenged RAW 264.7 cells significantly chemoattracted human MSCs, which can be inhibited by neutralizing antibody to MIP- $1 \alpha$. This result indicated that particlestimulated MIP- $1 \alpha$ release was responsible for the migration of MSCs [79]. CCR1 was one of the receptors for MIP- $1 \alpha$. Huang et al. found that neutralizing antibody to CCR1 failed to affect the migration of MSCs in culture with wear particles in vitro. On the contrary, using a murine model, Gibon et al. have recently shown that UHWMPE particles induced increased migration and osteoblast differentiation of MSCs in vivo, which can be neutralized by antagonist to CCR1 [82]. Since CCR 1 can bind to a variety of ligands including MIP$1 \alpha$, MCP-3, and RANTES, these findings were not sufficient to support the chemotaxis ability of MIP- $1 \alpha$. The specific chemokines participated in the migration of MSCs to the site around implants that need further investigation.

CCL17 and CCL22 are the two recognized ligands for the chemokine receptor CCR4. They are known to be mainly produced by cell lineages closely related to osteoclasts such as dendritic cells. CCL22 has been shown to be expressed by activated macrophages and mature dendritic cells, whereas CCL17 has been shown to be secreted by keratinocytes and endothelial cells. Titanium particles increased the expressions of CCL17 and CCL22 in osteoclasts and hFOB cells. Moreover, the expression of CCR4 was upregulated when osteoclast precursors were stimulated with titanium particles. These results implied a role for CCL17 and CCL22 in the chemotaxis of CCR4 expressing osteoclast progenitors to the site around implants [83].

\section{Conclusions and Perspective}

Aseptic loosening is the common cause of the failure of TJR, and the mechanisms underlying it appear complex and multifaceted. A large number of scholars have focused on the biological activity of wear particles. Macrophages recognize wear particles and release proinflammatory mediators, leading to osteoclast activation and osteolysis. Although it has been accepted that the interaction between wear particles and macrophages is critical, little is known about how wear particles are recognized and activate macrophages in the early inflammatory response. In vivo and in vitro studies have supplied strong evidence that wear particles can activate macrophages through TLRs. TLRs are evolutionarily conserved pattern recognition receptors in sensing exogenous PAMPs and endogenous DAMPs. Both PAMPs and DAMPs are responsible for the activation of TLRs in aseptic loosening. DAMPs/PAMPs-TLR interaction may be a novel mechanism of aseptic loosening. Although the relationship between TLRs and chemokines have not been clarified, it seems possible that the activation of TLR may promote macrophages to express different chemokines. In brief, the pathogenesis of implant-associated aseptic loosening includes a series of events: macrophages response to wear particles acting as PAMPs/DAMPs via various TLRs, especially TLR4 and TLR2. These TLRs then interact with adaptor protein MyD88, finally triggering the activation of NF- $\kappa \mathrm{B}$ and production of inflammatory cytokines. The cytokines induced by wear particles include chemokines such as IL-8, MCP-1, and MIP-1. These chemokines bind to specific G-protein-linked transmembrane receptors and activate intracellular signaling pathways, leading to recruit more macrophages, MSCs, neutrophils, and osteoclasts to the site of injury. The accumulation of cells further facilitates propagation of inflammatory and subsequent osteolytic events.

Given the fact that wear particles are recognized via TLRs, effective strategies can be designed to block the event. For example, MyD88 interfering may block TLR downstream signaling pathway and then prevent wear particle-induced periprosthetic osteolysis. Another promising approach involves inhibiting recruitment of macrophages and other cells to the inflammatory site. Pharmacologic intervention targeted at chemokine-receptor axis may provide the means to mitigate the response to wear particles. Indeed, in vivo study has shown disruption of MCP-1 ligand-receptor axis can inhibit wear particle-induced migration of macrophages and osteolysis. In general, understanding the mechanisms of wear particle-induced cellular activation and migration will provide insight into the prevention and treatment of prosthetic aseptic loosening. The future of research needs to focus on some areas: the specific TLRs which are activated by exposure to different types of wear particles, the downstream signaling pathway mediated by TLR, the specific chemokinereceptor axis participating in wear particle-induced cellular migration, the exact role of cell recruited by wear particles in aseptic loosening.

\section{References}

[1] A. Farber, R. Chin, Y. Song, P. Huie, and S. Goodman, "Chronic antigen-specific immune-system activation may potentially be involved in the loosening of cemented acetabular components," Journal of Biomedical Materials Research, vol. 55, no. 3, pp. 433-441, 2001.

[2] P. Koulouvaris, K. Ly, L. B. Ivashkiv et al., "Expression profiling reveals alternative macrophage activation and impaired osteogenesis in periprosthetic osteolysis," Journal of Orthopaedic Research, vol. 26, no. 1, pp. 106-116, 2008.

[3] R. Chiu, M. Ting, R. L. Smith, and S. B. Goodman, "Ultrahigh molecular weight polyethylene wear debris inhibits osteoprogenitor proliferation and differentiation in vitro," Journal of Biomedical Materials Research Part A, vol. 89, no. 1, pp. 242247, 2009.

[4] S. Kurtz, K. Ong, E. Lau, F. Mowat, and M. Halpern, "Projections of primary and revision hip and knee arthroplasty in the 
United States from 2005 to 2030," Journal of Bone and Joint Surgery Series A, vol. 89, no. 4, pp. 780-785, 2007.

[5] P. Herberts and H. Malchau, "Long-term registration has improved the quality of hip replacement: a review of the Swedish THR Register comparing 160,000 cases," Acta Orthopaedica Scandinavica, vol. 71, no. 2, pp. 111-121, 2000.

[6] O. Robertsson, K. Knutson, S. Lewold, and L. Lidgren, "The Swedish Knee Arthroplasty Register 1975-1997: an update with special emphasis on 41,223 knees operated on in 19881997," Acta Orthopaedica Scandinavica, vol. 72, no. 5, pp. 503513, 2001.

[7] E. Ingham and J. Fisher, "The role of macrophages in osteolysis of total joint replacement," Biomaterials, vol. 26, no. 11, pp. 1271-1286, 2005.

[8] S. Goodman, P. Aspenberg, Y. Song et al., "Tissue ingrowth and differentiation in the bone-harvest chamber in the presence of cobalt-chromium-alloy and high-density-polyethylene particles," Journal of Bone and Joint Surgery Series A, vol. 77, no. 7, pp. 1025-1035, 1995.

[9] W. J. Maloney and R. L. Smith, "Periprosthetic osteolysis in total hip arthroplasty: the role of particulate wear debris," Instructional Course Lectures, vol. 45, pp. 171-182, 1996.

[10] A. J. Rao, E. Gibon, T. Ma, Z. Yao, R. L. Smith, and S. B. Goodman, "Revision joint replacement, wear particles, and macrophage polarization," Acta Biomaterialia, vol. 8, no. 7, pp. 2815-2823, 2012.

[11] J. D. Witt and M. Swann, "Metal wear and tissue response in failed titanium alloy total hip replacements," Journal of Bone and Joint Surgery Series B, vol. 73, no. 4, pp. 559-563, 1991.

[12] P. H. Wooley and E. M. Schwarz, "Aseptic loosening," Gene Therapy, vol. 11, no. 4, pp. 402-407, 2004.

[13] P. E. Purdue, "Alternative macrophage activation in periprosthetic osteolysis," Autoimmunity, vol. 41, no. 3, pp. 212-217, 2008.

[14] K. Miyanishi, M. C. D. Trindade, T. Ma, S. B. Goodman, D. J. Schurman, and R. L. Smith, "Periprosthetic osteolysis: induction of vascular endothelial growth factor from human monocyte/macrophages by orthopaedic biomaterial particles," Journal of Bone and Mineral Research, vol. 18, no. 9, pp. 15731583, 2003.

[15] P. E. Purdue, P. Koulouvaris, B. J. Nestor, and T. P. Sculco, "The central role of wear debris in periprosthetic osteolysis," HSS Journal, vol. 2, no. 2, pp. 102-113, 2006.

[16] S. M. Horowitz and J. B. Gonzales, "Inflammatory response to implant particulates in a macrophage/osteoblast coculture model," Calcified Tissue International, vol. 59, no. 5, pp. 392396, 1996.

[17] C. A. St Pierre, M. Chan, Y. Iwakura, D. C. Ayers, E. A. Kurt-Jones, and R. W. Finberg, "Periprosthetic osteolysis: characterizing the innate immune response to titanium wearparticles," Journal of Orthopaedic Research, vol. 28, no. 11, pp. 1418-1424, 2010.

[18] R. T. Beck, K. D. Illingworth, and K. J. Saleh, "Review of periprosthetic osteolysis in total joint arthroplasty: an emphasis on host factors and future directions," Journal of Orthopaedic Research, vol. 30, no. 4, pp. 541-546, 2012.

[19] T. W. Bauer, "Particles and periimplant bone resorption," Clinical Orthopaedics and Related Research, no. 405, pp. 138143, 2002.

[20] T. Koreny, M. Tunyogi-Csapó, I. Gál, C. Vermes, J. J. Jacobs, and T. T. Glant, "The role of fibroblasts and fibroblast-derived factors in periprosthetic osteolysis," Arthritis and Rheumatism, vol. 54, no. 10, pp. 3221-3232, 2006.

[21] D. P. Pioletti and A. Kottelat, "The influence of wear particles in the expression of osteoclastogenesis factors by osteoblasts," Biomaterials, vol. 25, no. 27, pp. 5803-5808, 2004.

[22] C. C. Okafor, H. Haleem-Smith, P. Laqueriere, P. A. Manner, and R. S. Tuan, "Particulate endocytosis mediates biological responses of human mesenchymal stem cells to titanium wear debris," Journal of Orthopaedic Research, vol. 24, no. 3, pp. 461-473, 2006.

[23] M. L. Wang, L. J. Nesti, R. Tuli et al., "Titanium particles suppress expression of osteoblastic phenotype in human mesenchymal stem cells," Journal of Orthopaedic Research, vol. 20, no. 6, pp. 1175-1184, 2002.

[24] H. Haleem-Smith, E. Argintar, C. Bush et al., "Biological responses of human mesenchymal stem cells to titanium wear debris particles," Journal of Orthopaedic Research, vol. 30, no. 6, pp. 853-863, 2012.

[25] M. Takagi, Y. Tamaki, H. Hasegawa et al., "Toll-like receptors in the interface membrane around loosening total hip replacement implants," Journal of Biomedical Materials Research Part A, vol. 81, no. 4, pp. 1017-1026, 2007.

[26] T. Hirayama, Y. Tamaki, Y. Takakubo et al., "Toll-like receptors and their adaptors are regulated in macrophages after phagocytosis of lipopolysaccharide-coated titanium particles," Journal of Orthopaedic Research, vol. 29, no. 7, pp. 984-992, 2011.

[27] J. I. Pearl, T. Ma, A. R. Irani et al., "Role of the Toll-like receptor pathway in the recognition of orthopedic implant wear-debris particles," Biomaterials, vol. 32, no. 24, pp. 5535-5542, 2011.

[28] J. Lassus, V. Waris, J. W. Xu et al., "Increased interleukin-8 (IL-8) expression is related to aseptic loosening of total hip replacement," Archives of Orthopaedic and Trauma Surgery, vol. 120, no. 5-6, pp. 328-332, 2000.

[29] X. Mao, X. Pan, X. Peng, T. Cheng, and X. Zhang, "Inhibition of titanium particle-induced inflammation by the proteasome inhibitor bortezomib in murine macrophage-like RAW 264.7 cells," Inflammation, vol. 35, no. 4, pp. 1411-1418, 2012.

[30] M. Takagi, "Toll-like receptor-a potent driving force behind rheumatoid arthritis," Journal of Clinical and Experimental Hematopathology, vol. 51, no. 2, pp. 77-92, 2011.

[31] B. Lemaitre, E. Nicolas, L. Michaut, J. M. Reichhart, and J. A. Hoffmann, "The dorsoventral regulatory gene cassette spatzle/Toll/Cactus controls the potent antifungal response in Drosophila adults," Cell, vol. 86, no. 6, pp. 973-983, 1996.

[32] C. Pasare and R. Medzhitov, "Toll-like receptors: linking innate and adaptive immunity," Microbes and Infection, vol. 6, no. 15, pp. 1382-1387, 2004.

[33] R. M. Pope and Q. Q. Huang, "The role of Toll-like receptors in rheumatoid arthritis," Current Rheumatology Reports, vol. 11, no. 5, pp. 357-364, 2009.

[34] S. Akira and K. Takeda, "Toll-like receptor signalling," Nature Reviews Immunology, vol. 4, no. 7, pp. 499-511, 2004.

[35] K. Takeda and S. Akira, "Toll-like receptors in innate immunity," International Immunology, vol. 17, no. 1, pp. 1-14, 2005.

[36] G. Y. Chen and G. Nuñez, "Sterile inflammation: sensing and reacting to damage," Nature Reviews Immunology, vol. 10, no. 12, pp. 826-837, 2010.

[37] T. Kondo, T. Kawai, and S. Akira, "Dissecting negative regulation of Toll-like receptor signaling," Trends in Immunology, vol. 33, no. 9, pp. 449-458, 2012.

[38] J. Pajarinen, E. Cenni, L. Savarino et al., "Profile of toll-like receptor-positive cells in septic and aseptic loosening of total 
hip arthroplasty implants," Journal of Biomedical Materials Research Part A, vol. 94, no. 1, pp. 84-92, 2010.

[39] T. Lähdeoja, J. Pajarinen, V. P. Kouri, T. Sillat, J. Salo, and Y. T. Konttinen, "Toll-like receptors and aseptic loosening of hip endoprosthesis-a potential to respond against danger signals?" Journal of Orthopaedic Research, vol. 28, no. 2, pp. 184-190, 2010.

[40] H. N. Hao, B. Zheng, S. Nasser et al., "The roles of monocytic heat shock protein 60 and Toll-like receptors in the regional inflammation response to wear debris particles," Journal of Biomedical Materials Research Part A, vol. 92, no. 4, pp. 13731381, 2010.

[41] E. M. Greenfield, M. A. Beidelschies, J. M. Tatro, V. M. Goldberg, and A. G. Hise, "Bacterial pathogen-associated molecular patterns stimulate biological activity of orthopaedic wear particles by activating cognate toll-like receptors," The Journal of Biological Chemistry, vol. 285, no. 42, pp. 32378-32384, 2010.

[42] J. Pajarinen, Z. Mackiewicz, R. Pöllänen et al., "Titanium particles modulate expression of Toll-like receptor proteins," Journal of Biomedical Materials Research Part A, vol. 92, no. 4, pp. 1528-1537, 2010.

[43] Y. Tamaki, Y. Takakubo, K. Goto et al., "Increased expression of toll-like receptors in aseptic loose periprosthetic tissues and septic synovial membranes around total hip implants," Journal of Rheumatology, vol. 36, no. 3, pp. 598-608, 2009.

[44] H. Kumar, T. Kawai, and S. Akira, "Toll-like receptors and innate immunity," Biochemical and Biophysical Research Communications, vol. 388, no. 4, pp. 621-625, 2009.

[45] K. Ohashi, V. Burkart, S. Flohé, and H. Kolb, "Cutting edge: heat shock protein 60 is a putative endogenous ligand of the toll-like receptor-4 complex," The Journal of Immunology, vol. 164, no. 2, pp. 558-561, 2000.

[46] L. Hou, H. Sasaki, and P. Stashenko, "Toll-like receptor 4deficient mice have reduced bone destruction following mixed anaerobic infection," Infection and Immunity, vol. 68, no. 8, pp. 4681-4687, 2000.

[47] L. Zhuang, J. Y. Jung, E. W. Wang et al., "Pseudomonas aeruginosa lipopolysaccharide induces osteoclastogenesis through a toll-like receptor 4 mediated pathway in vitro and in vivo," Laryngoscope, vol. 117, no. 5, pp. 841-847, 2007.

[48] Y. Bi, J. M. Seabold, S. G. Kaar et al., "Adherent endotoxin on orthopedic wear particles stimulates cytokine production and osteoclast differentiation," Journal of Bone and Mineral Research, vol. 16, no. 11, pp. 2082-2091, 2001.

[49] J. L. Nalepka, M. J. Lee, M. J. Kraay et al., "Lipopolysaccharide found in aseptic loosening of patients with inflammatory arthritis," Clinical Orthopaedics and Related Research, no. 451, pp. 229-235, 2006.

[50] R. Maitra, C. C. Clement, B. Scharf et al., "Endosomal damage and TLR2 mediated inflammasome activation by alkane particles in the generation of aseptic osteolysis," Molecular Immunology, vol. 47, no. 2-3, pp. 175-184, 2009.

[51] B. J. Rollins, “Chemokines," Blood, vol. 90, no. 3, pp. 909-928, 1997.

[52] E. J. Fernandez and E. Lolis, "Structure, function, and inhibition of chemokines," Annual Review of Pharmacology and Toxicology, vol. 42, pp. 469-499, 2002.

[53] A. Zlotnik and O. Yoshie, "Chemokines: a new classification system and their role in immunity," Immunity, vol. 12, no. 2, pp. 121-127, 2000.

[54] J. E. Pease and T. J. Williams, "Chemokines and their receptors in allergic disease," Journal of Allergy and Clinical Immunology, vol. 118, no. 2, pp. 305-318, 2006.
[55] S. J. Allen, S. E. Crown, and T. M. Handel, "Chemokine: receptor structure, interactions, and antagonism," Annual Review of Immunology, vol. 25, pp. 787-820, 2007.

[56] M. Lind, M. C. D. Trindade, D. J. Schurman, S. B. Goodman, and R. L. Smith, "Monocyte migration inhibitory factor synthesis and gene expression in particle-activated macrophages," Cytokine, vol. 12, no. 7, pp. 909-913, 2000.

[57] Y. Nakashima, D. H. Sun, M. C. D. Trindade et al., "Induction of macrophage C-C chemokine expression by titanium alloy and bone cement particles," Journal of Bone and Joint Surgery Series B, vol. 81, no. 1, pp. 155-162, 1999.

[58] B. Yaszay, M. C. D. Trindade, M. Lind, S. B. Goodman, and R. L. Smith, "Fibroblast expression of C-C chemokines in response to orthopaedic biomaterial particle challenge in vitro," Journal of Orthopaedic Research, vol. 19, no. 5, pp. 970976, 2001.

[59] P. G. Ren, Z. Huang, T. Ma, S. Biswal, R. L. Smith, and S. B. Goodman, "Surveillance of systemic trafficking of macrophages induced by UHMWPE particles in nude mice by noninvasive imaging," Journal of Biomedical Materials Research Part A, vol. 94, no. 3, pp. 706-711, 2010.

[60] P. G. Ren, A. Irani, Z. Huang, T. Ma, S. Biswal, and S. B. Goodman, "Continuous infusion of UHMWPE particles induces increased bone macrophages and osteolysis," Clinical Orthopaedics and Related Research, vol. 469, no. 1, pp. 113122, 2011.

[61] D. G. Remick, “Interleukin-8," Critical Care Medicine, vol. 33, no. 12, supplement, pp. S466-S467, 2005.

[62] J. J. Rose, J. F. Foley, P. M. Murphy, and S. Venkatesan, "On the mechanism and significance of ligand-induced internalization of human neutrophil chemokine receptors CXCR1 and CXCR2," The Journal of Biological Chemistry, vol. 279, no. 23, pp. 24372-24386, 2004.

[63] D. J. J. Waugh and C. Wilson, "The interleukin-8 pathway in cancer," Clinical Cancer Research, vol. 14, no. 21, pp. 67356741, 2008.

[64] A. S. Shanbhag, J. J. Jacobs, J. Black, J. O. Galante, and T. T. Glant, "Cellular mediators secreted by interfacial membranes obtained at revision total hip arthroplasty," Journal of Arthroplasty, vol. 10, no. 4, pp. 498-506, 1995.

[65] A. Sabokbar and N. Rushton, "Role of inflammatory mediators and adhesion molecules in the pathogenesis of aseptic loosening in total hip arthroplasties," Journal of Arthroplasty, vol. 10, no. 6, pp. 810-816, 1995.

[66] E. A. Fritz, T. T. Glant, C. Vermes, J. J. Jacobs, and K. A. Roebuck, "Titanium particles induce the immediate early stress responsive chemokines IL- 8 and MCP-1 in osteoblasts," Journal of Orthopaedic Research, vol. 20, no. 3, pp. 490-498, 2002.

[67] E. A. Fritz, J. J. Jacobs, T. T. Glant, and K. A. Roebuck, "Chemokine IL- 8 induction by particulate wear debris in osteoblasts is mediated by NF- $\kappa \mathrm{B}$," Journal of Orthopaedic Research, vol. 23, no. 6, pp. 1249-1257, 2005.

[68] E. A. Fritz, T. T. Glant, C. Vermes, J. J. Jacobs, and K. A. Roebuck, "Chemokine gene activation in human bone marrow-derived osteoblasts following exposure to particulate wear debris," Journal of Biomedical Materials Research Part A, vol. 77, no. 1, pp. 192-201, 2006.

[69] K. Lochner, A. Fritsche, A. Jonitz et al., "The potential role of human osteoblasts for periprosthetic osteolysis following exposure to wear particles," International Journal of Molecular Medicine, vol. 28, no. 6, pp. 1055-1063, 2011.

[70] A. M. Kaufman, C. I. Alabre, H. E. Rubash, and A. S. Shanbhag, "Human macrophage response to UHMWPE, 
TiAlV, CoCr, and alumina particles: analysis of multiple cytokines using protein arrays," Journal of Biomedical Materials Research Part A, vol. 84, no. 2, pp. 464-474, 2008.

[71] A. J. Valente, D. T. Graves, C. E. Vialle-Valentin, R. Delgado, and C. J. Schwartz, "Purification of a monocyte chemotactic factor secreted by nonhuman primate vascular cells in culture," Biochemistry, vol. 27, no. 11, pp. 4162-4168, 1988.

[72] K. Matsushima, C. G. Larsen, G. C. DuBois, and J. J. Oppenheim, "Purification and characterization of a novel monocyte chemotactic and activating factor produced by a human myelomonocytic cell line," The Journal of Experimental Medicine, vol. 169, no. 4, pp. 1485-1490, 1989.

[73] I. F. Charo and M. B. Taubman, "Chemokines in the pathogenesis of vascular disease," Circulation Research, vol. 95, no. 9, pp. 858-866, 2004.

[74] F. Balkwill, "Cancer and the chemokine network," Nature Reviews Cancer, vol. 4, no. 7, pp. 540-550, 2004.

[75] Y. Lu, G. Xiao, D. L. Galson et al., "PTHrP-induced MCP1 production by human bone marrow endothelial cells and osteoblasts promotes osteoclast differentiation and prostate cancer cell proliferation and invasion in vitro," International Journal of Cancer, vol. 121, no. 4, pp. 724-733, 2007.

[76] X. Li, L. Qin, M. Bergenstock, L. M. Bevelock, D. V. Novack, and N. C. Partridge, "Parathyroid hormone stimulates osteoblastic expression of MCP-1 to recruit and increase the fusion of pre/osteoclasts," The Journal of Biological Chemistry, vol. 282, no. 45, pp. 33098-33106, 2007.

[77] M. C. D. Trindade, D. J. Schurman, W. J. Maloney, S. B. Goodman, and R. L. Smith, "G-protein activity requirement for polymethylmethacrylate and titanium particleinduced fibroblast interleukin-6 and monocyte chemoattractant protein-1 release in vitro," Journal of Biomedical Materials Research, vol. 51, no. 3, pp. 360-368, 2000.

[78] V. Dasa, J. M. Kramer, S. L. Gaffen, K. L. Kirkwood, and W. M. Mihalko, "Is monocyte chemotactic protein 1 elevated in aseptic loosening of TKA?: a pilot study," Clinical Orthopaedics and Related Research, vol. 470, no. 7, pp. 1879-1884, 2012.

[79] Z. Huang, T. Ma, P. G. Ren, R. L. Smith, and S. B. Goodman, "Effects of orthopedic polymer particles on chemotaxis of macrophages and mesenchymal stem cells," Journal of Biomedical Materials Research Part A, vol. 94, no. 4, pp. 1264-1269, 2010.

[80] C. D. L. Ramos, C. Canetti, J. T. Souto et al., "MIP- $1 \alpha$ [CCL3] acting on the CCR1 receptor mediates neutrophil migration in immune inflammation via sequential release of TNF- $\alpha$ and LTB4," Journal of Leukocyte Biology, vol. 78, no. 1, pp. 167-177, 2005.

[81] M. Maurer and E. Von Stebut, "Macrophage inflammatory protein-1," International Journal of Biochemistry and Cell Biology, vol. 36, no. 10, pp. 1882-1886, 2004.

[82] E. Gibon, T. Ma, P.-G. Ren et al., "Selective inhibition of the MCP-1-CCR2 ligand-receptor axis decreases systemic trafficking of macrophages in the presence of UHMWPE particles," Journal of Orthopaedic Research, vol. 30, no. 4, pp. 547-553, 2012.

[83] D. Cadosch, O. P. Gautschi, E. Chan, H. P. Simmen, and L. Filgueira, "Titanium induced production of chemokines CCL17/TARC and CCL22/MDC in human osteoclasts and osteoblasts," Journal of Biomedical Materials Research Part A, vol. 92, no. 2, pp. 475-483, 2010. 

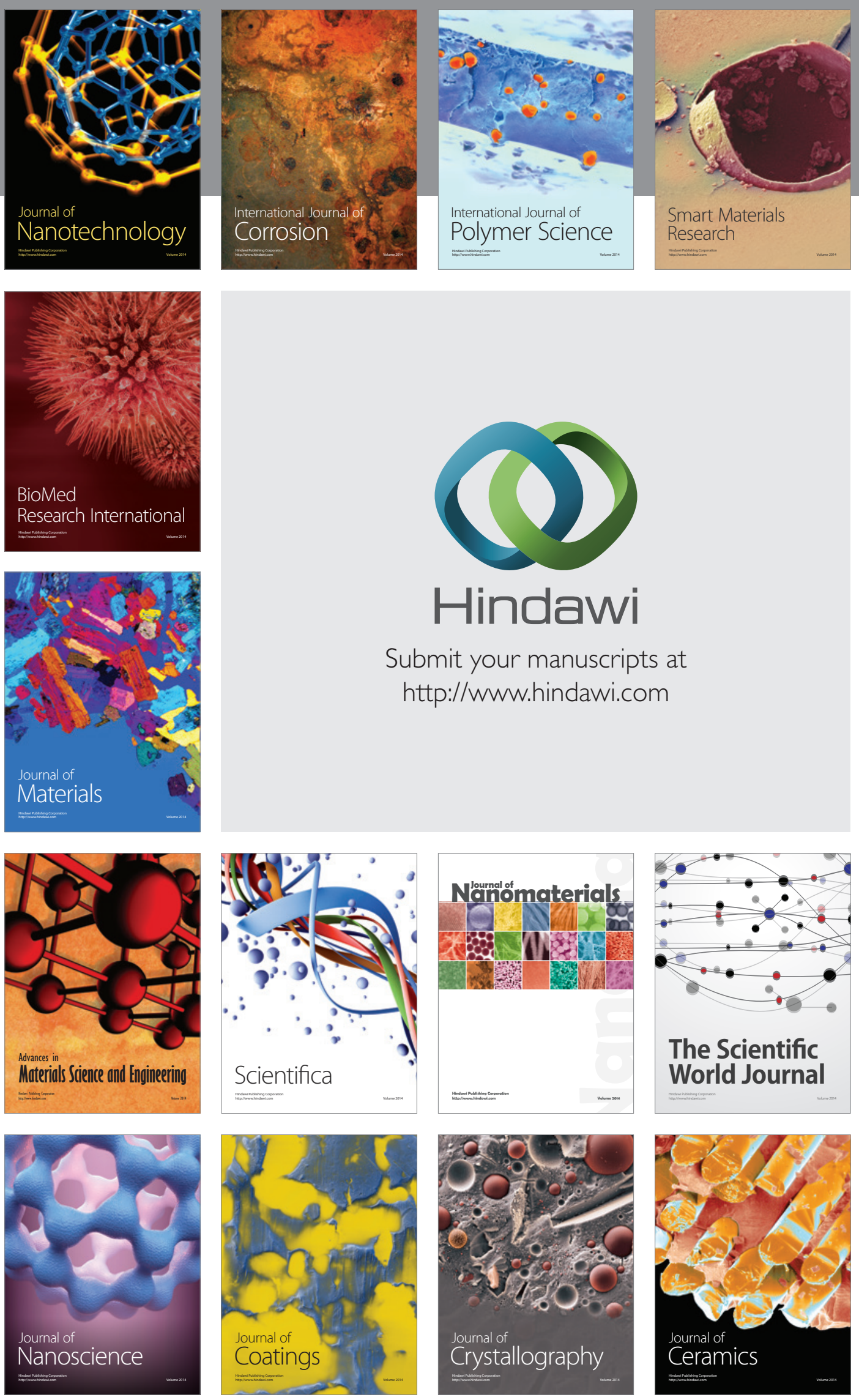

The Scientific World Journal

Submit your manuscripts at

http://www.hindawi.com

\section{World Journal}

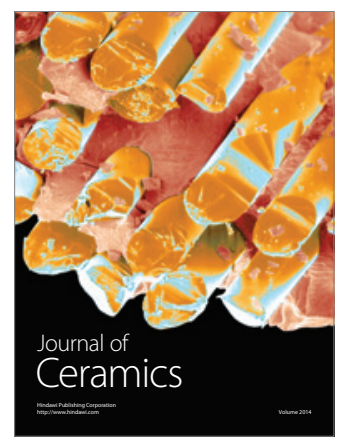

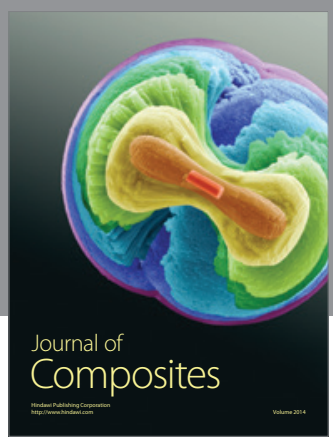
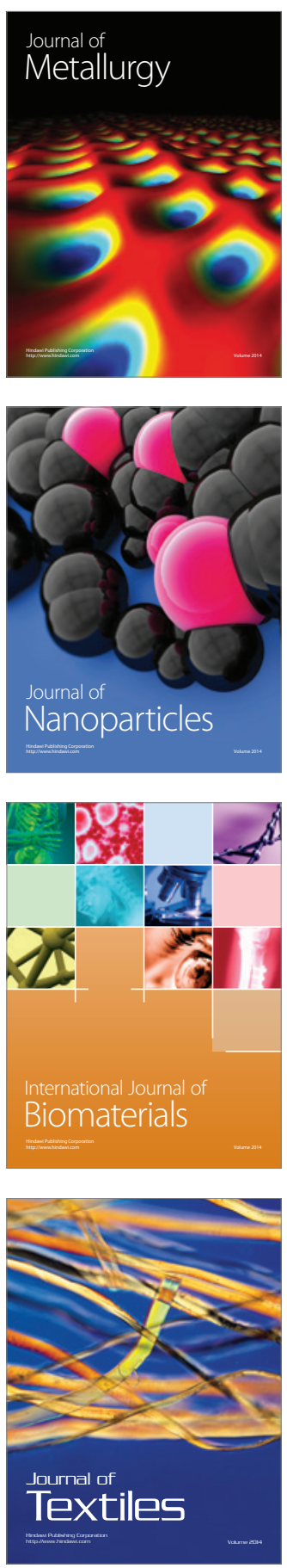